\title{
Bromberg in den Werken deutschsprachiger Schrift- steller. Autobiographische Reflexionen über die Stadt
}

\begin{abstract}
Artykuł przedstawia czterech wybranych niemieckich autorów, a na podstawie ich prozy autobiograficznej rolę Bydgoszczy w osobistej topografii pisarzy. Łącznikiem między nimi jest Bydgoszcz jako miejsce pewnego etapu życiowego oraz fakt pochodzenia $\mathrm{z}$ rodzin urzędniczych. Ich autobiografie są pretekstem do analizy wizerunku miasta.

Der Artikel stellt vier ausgewählte deutsche Autoren und anhand ihrer autobiographischen Prosa die Rolle der Stadt in der persönlichen Topographie der Schriftsteller dar. Was sie verbindet, sind Bromberg als ihr Wohnort und die Verknüpfung mit dem Schicksal der preußischen Beamtenfamilien. Ihre Autobiographien dienen hier nur als Vorwand und Mittel zur Stadtbildanalyse.

The article depicts four selected German authors and shows by their autobiographical prose the role of the city in the author's personal topographies. Their connection is Bromberg as their place of living and their linking to the faith of Prussian civil servant families. Their autobiographies are only a pretext and vehicle for the analysis of the image of Bromberg.
\end{abstract}

\section{Bydgoszcz entdeckt Bromberg}

Infolge der 1. Polnischen Teilung 1772 fiel Bromberg [Bydgoszcz] an Preußen und wurde seitdem beinahe 150 Jahre lang von preußischen Behörden verwaltet. Am 19. Februar 1807 fiel die Stadt an Polen zurück, ein Ergebnis der Errichtung des Großherzogtums Warschau. Nach dem Wiener Kongress von 1815 gehörte die Stadt wieder zu Preußen. Nach der Wiedererlangung der Unabhängigkeit Polens gehört sie seit dem 19. Januar 1920 zu Polen. Der Wissensstand der heutigen Bewohner von Bydgoszcz über ihren historischen All- 
tag ist lückenhaft. Dieser Zustand ist durch das Abbrechen der kollektiven Erinnerung entstanden. Seit einigen Jahren, nachdem die mentalen und politischen Hindernisse überwunden worden sind, fängt Bydgoszcz an, Bromberg zu entdecken. Das jahrzehntelang verdrängte preußische Kapitel der Stadtgeschichte ist heute Gegenstand vieler Diskussionen, was einen wichtigen Einfluss auf die Kenntnisse der Stadtgeschichte bei den Stadtbewohnern hat. Fast jedes Gebäude der heutigen Stadt Bydgoszcz erzählt eine Geschichte. Mit der Rekonstruktion dieser Geschichten befassen sich u.a. die Mitglieder der Gesellschaft der Liebhaber der Stadt. Die Ergebnisse ihrer Arbeit findet man in den periodischen Schriften des Vereins (Kronika Bydgoska ${ }^{1}$, Kalendarz Bydgoski ${ }^{2}$ ). Ihren Chefredakteuren ist es gelungen, eine große Gruppe von Forschern zur Mitarbeit zu gewinnen, dank denen immer neue Informationen aus der Vergangenheit die Rekonstruktion des alten und die Konstruktion des neuen Stadtbildes erleichtern. Die vergangene Geschichte dieser über 660 Jahre alten, zeitweilig recht bedeutenden Stadt, ist schon auf Grund ihrer bikulturellen Vergangenheit interessant, mehr noch, sie gewinnt ihren Reiz zurück und wird heute wiederbelebt. ${ }^{3}$ Ein sichtbares Zeichen sind auch Bildbände, unter ihnen einer von WOJCIECH BANACH (2001) Czas odnaleziony. Bydgoszcz na starej pocztówce 1894-1945, in dem eine Sammlung von ca. 500 reproduzierten Ansichtskarten aus Bromberg zu finden ist, und der neueste Bildband Piękna stara Bydgoszcz. (DERENDA 2007), der neben 800 Fotos noch preuBisch-deutsche und polnische Stadtkarten enthält. Doch allein die Aufnahmen von Bauwerken und Menschen erklären noch viel zu wenig aus dem Leben der

1 Die Kronika Bydgoska wird von Włodzimierz Jastrzębski herausgegeben. Mit ihm arbeiten zahlreiche Historiker zusammen. Die Jahresschrift reagiert auch auf die wichtigsten aktuellen lokalen Ereignisse. Ein Beweis kann die Sondernummer sein, die anlässlich des Besuches von Johannes Paul II. im Jahre 1999 in Bydgoszcz vorbereitet wurde.

2 Der Kalendarz Bydgoski erscheint seit 1968 aus Initiative der bekannten Journalistin Waleria Drygałowa. Er ist eine Jahresschrift, die der Stadt gewidmet ist und sie aus unterschiedlichen Perspektiven, einer historischen und einer aktuellen präsentiert. Unter den Autoren findet man Wissenschaftler, Journalisten und Liebhaber der Stadt. Es sind Beiträge, die nicht nur nach wissenschaftlichen Maßstäben geschrieben werden, sondern vielmehr auf populärwissenschaftliche Art und Weise den Reichtum des Bromberger Lebens damals und heute schildern.

3 Am 20.4.2006 ist im Leon-Wyczółkowski-Kreismuseum in Bromberg die Ausstellung „Polen und Deutsche in Bromberg“ eröffnet worden. Erschienen ist auch ein Heft mit demselben Titel (ISBN 83-86580-62-3), als Ergebnis einer Tagung, die am gleichen Tag stattgefunden hat. 
Stadt. Man möchte erfahren, wer die Bewohner waren, was sie dachten, was sie taten, wie ihr Alltag aussah (LOEW 2000:101f.). Momentan gibt es noch keine ausführliche polnische Publikation über die Vergangenheit Brombergs zu preußischen Zeiten, die versuchen würde, die Mentalität und Kultur seiner Bewohner aufzuzeigen. ${ }^{4}$ Vielversprechend ist deshalb die Möglichkeit, die gestellten Fragen anhand von literarischen Stadtkonstruktionen zu beantworten, die sich seit gewisser Zeit verstärkt im Zentrum der Aufmerksamkeit mancher Literaturforscher befinden. Die geschichtlichen, künstlerischen und literarischen Verknüpfungen einer Region, einer Stadt, sind häufig der Hintergrund von Erzählungen, Romanen und Novellen.

Bromberg hat jedoch im Gegensatz zu anderen Städten keinen Grass oder Janosch, nicht einmal seinen eigenen Caspar David Friedrich oder Otto Runge. Deswegen ist es heute nicht leicht, über die deutschsprachigen Bromberger, ihre literarischen oder künstlerischen Zeugnisse zu sprechen. Städte wie Bromberg müssen sich mit Künstlern von lokalem Rang zufriedengeben, die das Stadtbild mitgestalteten. Keiner von den Bromberger Dichtern und Schriftstellern wurde weltweit oder landesweit berühmt. Es ist trotzdem angebracht, auf das Werden der jeweiligen Region einzugehen, das mittels der Literatur wiederentdeckt und wahrgenommen werden kann.

Trotz des fast 150-jährigen preußischen Kapitels in der Geschichte der Stadt ist es schwierig, in Bezug auf Bromberg über Traditionen von deutschsprachiger Literatur, geschweige denn von literarischen Kreisen zu sprechen, hauptsächlich deswegen, weil das Deutschtum ein zugewandertes Element war und keine langjährige, literarische Tradition hat schaffen können. Bis auf den Salon der Familie Roquette, der in den 40er Jahren des 19. Jhd.s literarisch und publizistisch aktive Persönlichkeiten Brombergs und der Umgebung vereinigte (NOWIKIEWICZ 2002:302-312), ist es nicht gelungen, auf andere Beispiele einer engeren Zusammenarbeit literarisch talentierter Lokalgrößen im Bromberg des 19. und der ersten Jahrzehnte des 20. Jhd.s zu stoßen. ${ }^{5}$ Erst seit dem 7.6.1900 warb der neu gegründete Literarisch-dramatische Verein zu Bromberg um neue Mitglieder. Der Ostdeutschen Presse nach bezweckte der Verein die „Hebung der Interessen und des Verständnisses für Literatur und dramati-

4 Die Autoren des Bandes von MARIAN BISKUP (1999) beschäftigen sich mit der Rekonstruktion wichtiger historischer Ereignisse und Prozesse. Sie schildern auch ausgewählte Aspekte des kulturellen und gesellschaftlichen Lebens der Bewohner. Das Alltagsleben ist nicht Gegenstand ihrer Analyse.

5 Gesellschaftlich aktiv war noch die musikalisch talentierte Familie Löwe. 
sche Kunst durch Besprechung der laufenden literarischen Erscheinungen, durch Veranstaltung von Vorlesungen, Rezitationen und privaten wie öffentlichen Aufführungen, durch gegenseitigen Meinungsaustausch und geistige Unterstützung bei literarischen Arbeiten einzelner Mitglieder des Vereins bzw. auch begabter Anfänger auf diesem oder schauspielerischem Gebiete“ (135, 1900 ). Das Entstehen der Literarischen Abteilung in der „Deutschen Gesellschaft für Kunst und Wissenschaft“" im Jahre 1903 trug zur regeren Aktivität der literarisch interessierten Bromberger Bürger bei (SAMEL 1993:9f.). Es fällt aber nicht leicht, viele Dichter und Schriftsteller aufzuzählen, die man mit der Stadt verbinden könnte. Das Kriterium, dessen man sich hier am besten bedienen sollte, ist, Bromberg als ihre Geburtsstadt bzw. als Ort einer Lebensetappe anzusehen. Ihr schriftstellerisches Schaffen ließe sich dann als Literatur deutschsprachiger Bromberger bezeichnen, nicht unbedingt war das Literatur über Bromberg. Jedoch war der Ruf, den die meisten hiesigen Autoren erlangten, selten bedeutend genug, als dass ihre Namen in Werken der deutschen Literaturgeschichte zu finden wären. Es ist nur gelungen, in der Geschichte der neuesten Literatur von 1830 bis auf die Gegenwart (KURZ 1872) auf die Dichter zu stoßen, die wir als Bromberger Autoren bezeichnen könnten. Genannt seien an dieser Stelle Otto Roquette (1824-1896), Julie Pfannenschmidt (Ps. Julie Burow, 1806-1868), Albert Schulz (Ps. San Marte, 1802-1893) und Marie von Roskowska (1828-1889). Moderne Literaturgeschichten berichten auch nicht über die vielen Dichter und Schriftsteller des 18. und 19. Jhd.s, die nur im engen Bereich ihrer Heimat bekannt geworden sind. „Ihre Wirkung war dort oft groß, aber sie blieben eben ,Lokalgrößen““, stellte ELISABETH FRIEDRICHS (1967) in ihrer Abhandlung über die regionalen Literarischen Lokalgrößen 1700-1900 fest. Die Autorin bediente sich in ihrer Untersuchung der östlichen Provinzen Preußens, genauer gesagt, der hier interessierenden Provinz Posen ${ }^{6}$, eigentlich keiner Lexika, Register oder Verzeichnisse literarischer Lokalgrößen. Unter den 170 als Material zu dem Nachschlagewerk dienenden Texten beschäftigte sich nur der Artikel von PAUL LASKOWSKY (1927:178-188) ausschließlich mit der Provinz Posen. Friedrichs berücksichtigt bei ihrer Zusammenstellung nicht den für die Posener Literatur wichtigen Abdruck des Vortrags von GEORG MindE-PouET (1911:273-283), Die Provinz Posen in der modernen deutschen Dichtung ${ }^{7}$, und

6 Seit 1872 heißt das Großherzogtum Posen offiziell Provinz Posen mit zwei Regierungsbezirken, Posen und Bromberg. Geläufig waren auch die Bezeichnungen Posener Land oder Land Posen. Bis 1871 galt noch der Name Großherzogtum Posen.

7 Der Vortrag wurde auch im Posener Tageblatt vom 9. und 10.9.1910 abgedruckt. 
zahlreiche andere seiner Abhandlungen über die Literatur der Autoren aus der und über die Provinz Posen. Eine eingehende Untersuchung der deutschsprachigen, literarischen Provinz Posen blieb also erst heutigen Wissenschaftlern vorbehalten, die zahlreiche Biographien zusammenstellen konnten und die sogenannte Ostmarkenliteratur untersucht haben (POŁCZYŃSKA / WOJTCZAK 1996). Die Bromberger Autoren bleiben weiter im Schatten, obwohl es gelungen ist, eine Liste mit etwa zwanzig Namen zusammenzustellen, darunter: Anna Bolte (geb. 1852), Marie (Wilhelmine Emilie) Brandrup (1844/49?-1907), Isaak Herzberg, Theodor Gottlieb von Hippel (1857-?), Hermann Hoppe, Alfred Knobloch (1859-1916), Julius Kossarski (1812-1879), Franz Lüdtke (1882-1945), Waldemar Müller (1871-1955; seit 1908: MüllerEberhart), Clemens Conrad Rössler (1896-1980), Justus Rubehn, Hans von Saltzwedel, Bertha Wegner-Zell (1850-1927), Franz Werner (1862 - ?). Die meisten sind jedoch in Vergessenheit geraten, und ihre Lebensläufe lassen sich nur schwer rekonstruieren.

Nicht alle machten jedoch die Region zum Gegenstand ihrer literarischen Texte. Bromberger Motive sind zum Beispiel bei Herzberg, Knobloch, Kossarski, Lüdtke, Rössler, Rubehn, Werner präsent. Die Verbundenheit der Autoren mit dem Leben in der Region (mit der Stadt) wird auf den ersten Blick an deren Beschreibungen deutlich. Zur Regionalliteratur können somit noch literarische Texte überregionaler Autoren gerechnet werden, die das Milieu oder wichtige geschichtliche Ereignisse einer bestimmten Region zum Thema ihrer Romane und Erzählungen machen (ROSNER 1975:185-220). Recherchen haben einige Resultate erbracht. Es ist gelungen, umfangreichere Texte zu finden, die thematisch mit Bromberg verbunden sind. Da es nur wenige literarische Zeugnisse zum deutschsprachigen Schreiben über Bromberger regionale Erfahrungen gibt, ist es um so wichtiger, das zur Wahl stehende autobiographische Erzählen von vier deutschen Schriftstellerinnen und Schriftstellern, die als Bromberger Autoren angesehen werden können, zu berücksichtigen: JULIE BUROW (1857) ${ }^{8}$, OTTO ROQUETTE (1894) ${ }^{9}$, LEONTINE VON WINTERFELDPlaten (1957) und A.E. JoHANN (1989). Ganz typisch für fast alle Bromber-

\footnotetext{
So lautet ihr Geburts- und Künstlername.

9 Obwohl Otto Roquette im Alter von 15 Jahren die Stadt verlässt, lenkt die lokale Presse die Aufmerksamkeit der Leser auf sein weiteres Schicksal. Siehe: „Rubrik Literarisches“. In: Bromberger Wochenblatt 81 (1852); Ostdeutsche Presse 78/79 (1883); Otto Roquettes Beziehungen zu Bromberg. In: Ostdeutsche Rundschau 14/15 (1909); HANS MaCHATSCHECK (1930): Der Ostdeutsche Otto Roquette und das Bromberg seiner Zeit. In: Deutsche Schulzeitung in Polen 19/20:285-289.
} 
ger Schriftsteller ist die Tatsache, dass sie in ihrer Existenz als Schriftsteller noch einen anderen Beruf ausüben, meist den eines Hochschul- oder Gymnasialprofessors, eines Journalisten oder einer Lehrerin.

Der Versuch einer Selbstbiographie (1857) von Julie Burow (1806-1868) ist relativ kurz (84 Seiten) und umfasst die Lebensspanne von etwa dem dritten Lebensjahr bis ungefähr 1855. Die Ereignisse werden chronologisch präsentiert. Bildung und Stellung der Frau in der bürgerlichen Gesellschaft stehen hier wie in ihrem Gesamtwerk im Vordergrund. Somit vollzieht sich hier ein Stück Gesellschaftsgeschichte mit der bürgerlichen Frau im Vordergrund. Die Autobiographie von Otto Roquette (1824-1896) erschien 1894, am Lebensende des Dichters, und umfasst mit ihren zwei Bänden auf über 600 Seiten die Lebensspanne von der Kindheit bis etwa dem siebzigsten Lebensjahr (1892) des erzählenden Ichs, wovon der Schriftsteller die Zeit von 1833 bis etwa 1839 in Bromberg verbringt. Roquette hält sich eng an die Chronologie der Ereignisse, was ein vorwiegend lineares Erzählen zur Folge hat. Diese Erzählweise erinnert an den Realismus des 19. Jhd.s. Vor dem Hintergrund der Autobiographie zeichnen sich nur wenige wirtschaftliche, gesellschaftliche oder politische Umbrüche ab (u.a. der Konflikt zwischen Österreich und Preußen im Jahre 1866). Vielmehr geht es um einen Lebensbericht, in dem vor allem das Private im Vordergrund steht. Die Lebenserinnerungen von Leontine von WinterfeldPlaten (1883-1960) erscheinen im Jahre 1957, drei Jahre vor ihrem Tod. Die autobiographische Erzählung Einer Mutter Kreuz erscheint früher, im Jahre 1949, kurz nach dem Zweiten Weltkrieg. Ein Beweis für die autobiographischen Züge dieses Buches ist vor allem das Vorwort der Autorin zur Buchausgabe der Lebenserinnerungen, die neben dem Teil 1 Zwischen Sonne und Sturm (1957) auch als zweiten Teil Einer Mutter Kreuz von 1949 enthält. Leontine von Winterfeld-Platen schreibt einleitend, dass Einer Mutter Kreuz vieles aus ihrem eigenen Leben enthalte, was sie im ersten Teil des Bandes nicht noch einmal wiederholen wollte, deswegen scheine es ihr und dem Verlag angebracht, die beiden Arbeiten zu vereinigen. Beide Teile sind somit ein Stück des Lebens der Schriftstellerin. Der erste Teil der Lebenserinnerungen umfasst auf 57 Seiten die Lebensspanne von der Kindheit bis zum Tod ihres Mannes (Herbst 1957), wobei der Schwerpunkt auf die Darstellung ihres privaten familiären Lebens fällt. Die Ich-Erzählerin schildert die wichtigsten Tatsachen aus ihrem Leben. Sie verfährt dabei sehr sachlich, es ist eher ein Bericht als eine Erzählung. Dadurch unterscheidet sich der erste Teil wesentlich vom zweiten, früher erschienenen (Einer Mutter Kreuz), der nicht sachlichberichtend verfasst ist, sondern emotionsgeladene Erinnerungsbilder enthält. Leiderfahrung und Leidthematisierung stehen in engem Zusammenhang. Sie 
beginnt den Rückblick mit dem Sommer 1939 und beendet die Geschichte Weihnachten 1945. Die Handlung findet dann in der Realität des Zweiten Weltkriegs statt. In Dies wilde Jahrhundert findet der Leser A.E. Johanns (1901-1996) persönliche Erlebnisse aus Kindheit, Jugend und Alter. Der Autor liefert mit diesem Buch weniger die Rekonstruktion des eigenen Lebens, sondern vielmehr den Rückblick auf das zu seinen Lebzeiten vergehende 20. Jhd. und den Einfluss dieser Erfahrungen auf ihn. Es ist ein Resümee und ein kritisches Urteil über eine Zeit und eine Welt, die er beobachtet und erlebt hat. Es sind episodische Überlegungen eines reifen Journalisten, der zu historischen, gesellschaftlichen, politischen und Glaubensfragen Stellung nimmt. Diese Bemerkungen verraten seinen außergewöhnlichen Sinn für Entwicklungstendenzen in der Welt. Somit ist ein beträchtlicher Teil seines Buches ein Bericht über das weltweit Gesehene und Erlebte (der Untergang des Kaiserreiches, das Hitler-Regime, Zweiter Weltkrieg, die Atombombe, der Untergang Deutschlands und Japans, die unmittelbare Nachkriegszeit mit Erfahrungen als Deutscher in England und Irland). Gleichzeitig ist ein großer Teil der Beiträge voller selbstbiographischer Elemente: 1. Herkunft und Kindheit in Bromberg, 2. Leitung einer naturwissenschaftlichen Berliner Monatsschrift, 3. Aufenthalt im US-amerikanischen Vernehmungslager und Arbeit im Redaktionsteam einer Lagerzeitung, 4. Leben ,auf Reisen“, das zu seiner zweiten Natur wird: Reise durch Amerika, Aufenthalt in Kanada (seinem Lieblingsland) und Irlandfahrt. In diesem autobiographischen Mosaik, in dem die Chronologie der Ereignisse nicht eingehalten wird, reflektiert der IchErzähler, der mit dem Autor identisch zu sein scheint, u. a. auch über seine Zeit in Bromberg bis etwa 1918. Im Unterschied zu den rein fiktionalen Gattungen wird in den nicht fiktionalen Werken die Wahrheit der Dichtung vorangestellt, es wird direkt auf die historische Realität eingegangen. Die Tatsache, dass der objektiven Tatsachendarstellung eine subjektive Erzählerposition gegenübergestellt wird, bereichert die Aussagekraft des autobiographischen Berichts, wodurch die transportierten Bilder authentischer erscheinen. Der Erzähler reflektiert über die Welt, in der er lebt. Wenn die Welt, in der er lebt, dem Leser vertraut ist, ist das, was jenseits der äußeren Handlung liegt, umso wichtiger: die Reflexionen über und Erinnerungen an diesen Ort. Die genannten Autoren verbringen schließlich nur ein paar Jahre ihres Lebens in Bromberg. Julie Burow kommt Mitte der 50er Jahre an und stirbt 1868. Otto Roquette ist uns als Bromberger Gymnasiast bekannt, der die Zeit von 1833 bis 1839 in Bromberg verbringt. Leontine von Winterfeld-Platen besucht in der Stadt das Lehrerinnenseminar und wohnt dort etwa in der Zeit von 1901 bis 
1908. A.E. Johann ist als einziger in Bromberg geboren und verbringt in der Stadt ungefähr die ersten siebzehn Jahre seines Lebens.

Die Präsenz der Stadt in der persönlichen Topographie der behandelten Schriftsteller ist von einer Reihe von Faktoren bestimmt, die teilweise einige biographische Details erläutern mögen. Julie Burow, am 24.2.1806 in Kudyllen (Ostpreußen) geboren, ist heute eine vergessene Autorin. Ihre Romane werden nicht neu aufgelegt. Sie verbringt ihre Jugend in Elbing und Tilsit, wo der Vater beim Zollwesen angestellt ist. Sie wird Erzieherin in Pohiebels bei Rastenburg/Masuren in Ostpreußen. Schließlich leben die Eltern in Danzig, wo ihr Vater eine Stelle als Regierungssekretär erhält. Die Stellung ihrer Eltern und ihr Bildungsgrad war von der Art, dass er den Eintritt in die bessere Gesellschaft ermöglichte (BUROW 1857:57). Die Familie lebt jedoch oft aufgrund des verschwenderischen Lebensstils des Vaters in finanzieller Not. Im Jahre 1831 heiratet Julie den Baumeister Pfannenschmidt aus Bromberg, der als Beamter seinen Arbeitsverpflichtungen nachgehen muss und somit zu Hause ein seltener Gast ist. Bei ihm führt sie ein stilles häusliches Leben an verschiedenen Orten. Sie zieht mit ihrem Gatten nach Neufahrwasser, einem Hafenstädtchen, eine Meile von Danzig entfernt, wo er einen Arbeitsstandort hatte. Von dort ziehen sie nach Pelplin. Der nächste Ort ist über ein Jahr lang Dirschau, dann Driesen in der Neumark (Wasserbaumeisterstelle) und Züllichau (hier ist ihr Mann als Landbauinspektor tätig). Letztendlich gelangt das Ehepaar nach Bromberg (um 1854), wo Julie Burow am 20. Februar 1868 stirbt. Das erste Buch von Julie Burow ist Frauenlos (1850). In ihren sehr zahlreichen Romanen und Novellen, die teilweise in Buchreihen - Bibliothek deutscher Romane, Prag: Kober und Markgraf; Hausbibliothek der Jugend, Berlin: Haffelberg - erscheinen, schildert sie mit viel Menschenkenntnis hauptsächlich das kleinbürgerliche Leben und die Zustände in preußischen Provinzstädten in der Mitte des 19. Jhd.s.

Otto Roquette, Dichter und Hochschullehrer, stammt aus einer französischen Flüchtlingsfamilie, die, aus Frankreich vertrieben, zuerst in die Pfalz und dann nach Brandenburg übersiedelte. Sein Großvater lebte als Pfarrer der französisch-reformierten Gemeinde in Frankfurt/Oder, und der Vater, Louis Jean Roquette, verheiratet mit Marie Antoinette Barraud, einer künstlerisch und geistig interessierten Frau, wird 1822 als Gerichtsassessor nach Krotoschin (Provinz Posen) versetzt. Hier kommt Otto am 19.4.1824 zur Welt. Louis Jean Roquette, inzwischen zum Landgerichtsrat ernannt, lässt sich dann als Justizkommissarius versetzen und kommt nach vorübergehender Tätigkeit in Gnesen gegen Ostern des Jahres 1833 nach Bromberg. Otto Roquette wechselt genauso 
häufig die Wohnorte wie seine Eltern. Nachdem er nach dem Abitur Frankfurt an der Oder verlässt, studiert er ab 1845 in Berlin, Heidelberg und Halle Philosophie, Geschichte und neue Sprachen. In der Hallenser Zeit (Ostern 18491851) erscheinen Roquettes erste Werke. Seine Waldeinsamkeit wird im Mai 1850 bei der Eröffnung des Berliner Friedrich-Wilhelmstädtischen Theaters gespielt. Bald wird das Stück auf vielen deutschen Bühnen aufgeführt, und der Einakter Die Probepredigt wird auch in Bromberg aufgeführt. In Heidelberg arbeitet er an Waldmeisters Brautfahrt (1851, darin das volkstümliche Lied Noch sind die Tage der Rosen). Dieses lyrisch-epische Werk ist ein bedeutender buchhändlerischer Erfolg. Letztendlich lässt sich Roquette in Darmstadt als Hochschullehrer nieder, wo er am 18.3.1896 stirbt. Nach ihm wird eine der dortigen Straßen benannt.

Leontine von Winterfeld-Platen wurde auf dem Gut ihres Urgroßvaters am 21.7.1883 zu Lübberstorf bei Friedland/Mecklenburg Strelitz geboren und entstammt einer sehr alten mecklenburgischen Adelsfamilie (BRUN 2005:1). Im Alter von 14 Jahren geht sie für zwei Jahre in Frankfurt/Oder zur Schule. Im Jahre 1901 ziehen ihre Eltern nach Bromberg, wo sie sich in den Jahren 19051908 zur Lehrerin ausbilden lässt. Nach der erfolgreich abgelegten staatlichen Prüfung übernimmt sie die Stelle einer Erzieherin im Haus des Grafen Dönhoff auf Friedrichstein bei Lömenhagen (Ostpreußen), die sie mindestens bis 1911 innehat. Sie heiratet im Dezember 1912 und lässt sich mit ihrem Gatten, Rittmeister von Platen, in Ludwigslust nieder. Zu dieser Zeit ist sie schon schriftstellerisch tätig. Ihr erstes Buch, Um der Menge schreien, wird 1910 verlegt, und ihr erstes Schauspiel in vier Akten, Sigelind (1913), findet seine Uraufführung im Bromberger Stadttheater im Februar 1914. Kurz danach bricht der Erste Weltkrieg aus und von Winterfeld-Platens Mann rückt ins Feld. Infolge des Krieges muss die Familie ihren Wohnsitz nach Krüden verlegen. 1925 wird Krüden verkauft, und Leontine zieht mit ihrer Familie nach Neu Pinnow bei Warnow in der Westprignitz. Hier entsteht ein Großteil ihrer Bücher, hier stirbt sie am 24.7.1960. Als Soldaten- und später Beamtenfrau hat sie den Wohnsitz oft wechseln müssen, und somit schreibt sie in den Erinnerungen auch von immer neuen „Heimaten“. Sie war nie an einen Ort gefesselt, sie wollte es auch nicht, denn sie wusste, dass keiner dieser Orte der letzte ist.

Auch Alfred Ernst Johann Wollschläger, bekannt als A.E. Johann - neben Richard Katz und Colin Ross der erfolgreichste deutschsprachige Reiseschriftsteller des 20. Jhd.s - ist ein Beamtenkind. Er kommt am 3.9.1901 in Bromberg zur Welt und stirbt am 8.10.1996 im Heidedorf Oerrel in der Lüneburger Heide. In seiner Geburtsstadt absolviert er das Realgymnasium; im Ersten 
Weltkrieg ist er Kriegsfreiwilliger. Nach dem Ersten Weltkrieg ziehen seine Eltern nach Potsdam. Der Vater bleibt ,mittlerer“ Beamter, obwohl er sich, so der Ich-Erzähler, für den „höheren Dienst“ (JOHANN 1989:137) qualifiziert hatte. Die Gründe für die nicht erfolgte Beförderung seines Vaters sieht der Schriftsteller in des Vaters politischen Überzeugungen. Er befürwortete weder die Politik Bismarcks noch die sozialen und gesellschaftlichen Schichtungen im Kaiserreich. Erst in der Zeit der Weimarer Republik, als die Eliten der früheren Jahrhunderte keine Bedeutung mehr hatten, ,als die alten ,Standes '-Vorstellungen mit dem Abgang des Kaisers ins Schwimmen gerieten“ (JOHANN 1989:138), kommt die Chance für den Aufstieg. Wollschläger studiert inzwischen in Berlin Theologie, Geographie und Soziologie. Nach einer Banklehre gibt er 1927 seinen Beruf auf, da ihn die Reiselust überfällt. Er tritt nicht in die Fußstapfen des Vaters und ist ,nie der Versuchung erlegen, der Sicherheit vor der Unabhängigkeit den Vorrang einzuräumen“ (JOHANN 1989:141). Als landwirtschaftlicher Arbeiter wandert er nach Kanada aus. Von dort schickt Wollschläger seine ersten Reiseberichte an die Vossische Zeitung. Nun wird die Reiseschriftstellerei sein Lebensinhalt. Er ernährt sich vom Schreiben (JOHANN 1989:159). Er bevorzugt es, mit öffentlichen Verkehrsmitteln zu reisen und lernt deshalb Land und Leute besonders gut kennen. Einfallsreichtum kennzeichnen sowohl seine Reisen selbst als auch die Bücher, die er darüber schreibt. ${ }^{10}$ Als er nach Berlin zurückkehrt, schlägt ihm der Ullstein Verlag vor, aus seinen anschaulichen Berichten ein Buch zu machen. 1928 erscheint Mit 20 Dollar in den Wilden Westen, das bereits im ersten Jahr eine Auflage von über 150.000 Exemplaren erreicht. Schnell hört Johann auch auf, an die Beständigkeit der europäischen Mächte (deutsche, französische, britische) zu glauben. Schon 1927 unternimmt er seine erste Reise, um am jeweiligen Ort die Prozesse zu beobachten, die ihre Niederlage bewirkten. Er schlussfolgerte im Zusammenhang damit:

Große Reiche, ganz gleich welcher Art, sind nicht für die Ewigkeit geschaffen, sind verglichen mit den Zeitspannen der Geschichte, nur Eintagsfliegen, und es empfiehlt sich für den Feld-, Wald- und Wiesen-Einzelnen, alle diese politischen Gebilde mit großer Skepsis zu betrachten, sie , kühl bis ans Herz hinan' nach ihrer praktischen Leistungsfähigkeit zu beurteilen, sich ihnen von innen her niemals gläubig auszuliefern. (JOHANN 1989:196)

10 Neben Reisebeschreibungen verfasste Johann mehrere Romane, die meist in fernen Ländern spielen. Er war auch als Übersetzer tätig. Johann hat über 80 Buchmanuskripte verfasst, unzählige Artikel in Zeitungen und Zeitschriften veröffentlicht (u.a. in der Wochenzeitung Die Zeit, Westermanns Monatsheften, Merian Heften, Bertelsmann drei etc.). 
Der Prozess des Umdenkens beginnt bei ihm unmittelbar nach dem Ende des Ersten Weltkrieges vor dem Bromberger Stadttheater, nachdem der Kaiser geflohen und die Kaiserregierung zurückgetreten ist. Die Anhänger der Revolution versuchen, Soldaten und Zivilisten für ihre Ideen zu gewinnen. In jenem Moment begreift er, dass ,die großen Reiche sang- und klanglos untergehen früher oder später ein jedes dieser Reiche“ (JOHANN 1989:196). Sein Bedarf an Revolution ist damals völlig gedeckt, ebenso wie sein Bedarf an „Kaiser und Reich“, der in der Schule und auch sonst bis dahin nachhaltig genährt worden war (JoHANN 1989:195f.). Der Protagonist behauptet demzufolge, dass Menschen, ohne gefragt zu werden, in einen konkreten Staat hineingeboren werden. Und wenn sie an ihn als etwas Absolutes glauben, „handelt es sich nun um Kaiser, König oder Republik, um Diktatur oder ,Volksherrschaft ““ (JOHANN 1989:196), werden sie enttäuscht werden. Es darf somit geschlussfolgert werden, dass die Konfrontation der Jugendideale mit der Rolle des Feldsoldaten im Ersten Weltkrieg und schließlich der Zusammenbruch des deutschen Kaiserreiches bei ihm eine Art Schock verursacht haben müssen. Der von Wollschläger eingeschlagene Weg des Globetrotters scheint eine bessere Möglichkeit zu sein als die Abhängigkeit von irgendeinem Staatsgebilde, das bekanntlich, so der Ich-Erzähler, nicht beständig sein kann, denn der Untergang ist seine Natur. Man hat den Eindruck, dass sich der Ich-Erzähler mit abstrakten Staatsgebilden nicht mehr identifizieren will. Das bedeutet aber nicht, dass ihn das Schicksal der Deutschen, die aus den Gebieten weit jenseits von Oder und Neiße stammen, nicht bewegt (JOHANN 1989:202). Es geht hier keineswegs um die Sehnsucht nach dem Kaiserreich, sondern um die Zerstörung einer sicheren Oase (des Zuhauses, des Elternhauses), unabhängig davon, wo sie sich befindet. Trotzdem ist nur einmal vom „Verlust der Heimat" die Rede, als der Ich-Erzähler von seiner Freundschaft mit Hans, die selbst lange Trennung überdauert hatte, schreibt. Er meint, dass die beiden „inzwischen auf ganz verschiedene Weise vom Krieg, dem Verlust der Heimat und dem Nachkrieg mit seiner unheimlich rasch ansteigenden Inflation gebeutelt worden waren“ (JOHANN 1989:326). Der Erzähler leidet an den Folgen schwerer Erlebnisse. Er stellt sich eine ganze Reihe von Fragen, die jedoch unbeantwortet bleiben:

Wer bin ich, wo komme ich her, wo will ich hin, was ist der Sinn dieses gräßlichen Durcheinanders, das sich Geschichte nennt, warum ist so vieles schlecht, so wenig vernünftig und einleuchtend von dem, was rings umher vor sich geht, warum geschieht, um es neutestamentlich auszudrücken, das Gute nicht, das wir wollen, sondern das Böse, das wir nicht wollen? (JoHANN 1989:328f.) 
Der häufige Wechsel seiner Aufenthaltsorte und die Weltreisen sind schließlich sein Lebensinhalt. Er hängt weder an Bromberg als seinem Kindheitsort noch ist er mit einem anderen enger verbunden. Eine besondere Rolle spielt nur Kanada. Bromberg genießt keine privilegierte Stellung in seiner persönlichen Topographie. Er bleibt schließlich ein von Kanada faszinierter Weltbürger, der von Erfindungen und Entdeckungen der Menschheit, die das Weltgeschehen beeinflussen, begeistert ist. Für die wichtigste und verhängnisvollste Erfindung hält er die Atombombe.

Die behandelten Autoren sind somit keine repräsentativen deutschsprachigen Bromberger, denn sie sind Vertreter ein und derselben Gesellschaftsschicht (Beamte bzw. ihre Familien). Sie sind ähnlicher Abstammung und vergleichbar ausgebildet, und ihr Schicksal ist durch häufige Versetzungen geprägt. Daher ist die Fluktuation unter Beamten eine recht häufige Erscheinung. Dadurch, dass alle Autorinnen und Autoren in ihren Autobiographien häufige Versetzungen erwähnen, unterstreichen sie indirekt ihre zufällige Bindung an Bromberg. Eine emotionale Bindung an diesen Raum findet eigentlich nicht statt. Es ist eher eine Erinnerung an das Gefühl der Geborgenheit, an das Elternhaus, an die Sehnsüchte nach anderen Menschen, mit denen man gelebt hat. Bromberg ist in allen Fällen nur eine Übergangsstation, auch wenn man an A. E. Johann denkt, der als einziger hier geboren wurde. Versetzungen waren das Schicksal und die Wahl ihrer Eltern bzw. Lebensgenossen. Keiner von den Schriftstellern stellt fest, dass er sich hier heimischer fühlt als woanders. Bessere berufliche und künstlerische Möglichkeiten sehen sie für sich hier auch nicht. Als Bewohner stehen sie schließlich Bromberg eher gleichgültig gegenüber, obwohl schon die oberflächliche Lektüre dieser autobiographisch geprägten Bücher die allererste vorsichtige Schlussfolgerung erlaubt: Für alle vier Autobiographen ist die Häufigkeit des Nachdenkens über ihr Verhältnis zu der Stadt Bromberg mit der Bedeutung des Ortes in ihrem Leben gleichzusetzen. Erinnerungen an die Kindheit und Jugendzeit sind in der Regel lebendiger und farbiger. Es wundert nicht, dass Otto Roquette und A.E. Johann dem Bromberger Kapitel ihres Lebens mehr Platz einräumen als Julie Burow und Leontine von Winterfeld-Platen. Die Art und Weise, wie die besagten Schriftstellerinnen und Schriftsteller ihre Vergangenheit darstellen, lässt sich nicht mit dem Erinnern vieler Autorinnen und Autoren der Nachkriegszeit an die ostdeutsche Heimat ihrer Kindheit vergleichen. Hier geht es ausschließlich um Lust am Erinnern und nicht um Angst vor dem Vergessen oder um Sehnsucht nach dem Verlorenen. In Dies wilde Jahrhundert ist demzufolge zu lesen: „Heimat war sie mir und meinen Eltern nicht“ (JOHANN 1989:139), mehr noch: Er bedient sich in Bezug auf die Beamtenlaufbahn seines Vaters in der deut- 
schen Ostmark sogar der Formulierung „ungeliebter Dienst“ (JOHANN 1989: 138), was sich unterschiedlich interpretieren lässt:

- dass der Beamtendienst ,im fernen Osten des Reiches“ (JoHANn 1989:191) nicht leicht war; er schreibt aber nicht, was ihn so schwer machte;

- dass der Beamtendienst für geringes Gehalt mit kümmerlichem Urlaub (JOHANN 1989:138) nicht attraktiv genug war. Außerdem gab diese Arbeit keine Genugtuung, denn der berufliche Aufstieg war für Durchschnittsbürger (er stammte von Bauern und Handwerkern ab - JOHANN 1989:100, 140) nur auf recht umständlichem Weg möglich, während die adelige Abstammung die Aufnahme in die höhere Laufbahn ohne größere Hindernisse ermöglichte.

Soziale und gesellschaftliche Schichtungen waren damals noch zu stark. Das wusste sein Ich-Erzähler von Kindheit an:

,Vorgesetzter" war ein Wort, dessen Bedeutung mir schon früh aufgegangen war. Wenn sich also der Herr Geheimrat uns auf einige Schritte Abstand genähert hatte und mein Vater sich anschickte, den Hut zum respektvollen Gruß zu ziehen, dann griff ich nach meiner Mütze [...] und schwang sie mit einem ganz kleinen Diener. (JOHANN 1989:139f.)

Die inhaltliche Analyse der genannten Texte beweist auch, dass von negativen Urteilen nicht gesprochen werden kann. Die Autobiographen teilen nicht die allgemeine negative und stereotype Haltung bezüglich des geistigen und kulturellen Lebens in Bromberg in der angegebenen Zeit. Ihre Bemerkungen sind eher von universalem Charakter und beziehen sich auf bestimmte existentielle Fragen.

\section{Die Stadt als autobiographisches Motiv}

Trotz der oben erwähnten Einschränkungen verzichten die Autoren in ihren Autobiographien nicht auf Bromberger Motive. Die Stadt bildet jedoch keinen Handlungsrahmen, und die Sättigung der Autobiographien mit Bromberger Motiven ist nicht gleichmäßig. Otto Roquette und A.E. Johann sind an der Stadtbilddarstellung mehr interessiert als Burow und Leontine von WinterfeldPlaten. In der autobiographischen Lektüre von Julie Burow ist die Bromberger Zeit nur ein wenig konkreter Lebensabschnitt. Der Stadt wird keine besondere Bedeutung zugeschrieben. Beide Autorinnen konzentrieren sich fast ausschließlich auf ihr persönliches Leben. Burow schreibt von der Einsamkeit, ihren depressiven Zuständen, ihren Bedürfnissen und Sehnsüchten. Die Orte, in denen sie sich aufhält, spielen eine geringe Rolle. Was zählt, sind ihr Mann, 
Kinder, Familie, Nachbarn und nicht die Adresse, wo sie sich gerade aufhält. Als Bestätigung können folgende Zitate gelten: „Ich hatte ja keine Heimat! Ich hatte nur in der Ferne Herzen, nach denen ich mich sehnte!" (BUROw 1857:52) oder: „Die Wurzeln meines Lebensbaumes sollten nie zu fest sich einranken in eine irdische Heimat, - damit, so glaube ich - die Krone desselben desto freier emporsteige in den ewigen Aether" (BUROw 1857:40). Die Familie wird mit Heimat gleichgesetzt. Man kann lesen: ,[...] aber es war eine Heimat, es war ja doch ein Vaterhaus und das Familienband umschlug die vier Menschenherzen, die sie theilten" (BUROW 1857:55). Im Januar 1856 feiert Familie Pfannenschmidt in Bromberg den 25. Jahrestag ihrer Ehe. Herr Pfannenschmidt leitet damals in der Stadt den Bau einer neuen Realschule und der Post. Da er in ihren Aufenthaltsorten sichtbare Spuren seiner beruflichen Arbeit hinterlässt, will auch Julie im Gedächtnis der Bromberger Mitbewohner weniger als Schriftstellerin, sondern als Frau und Mutter, die das Haus pflegt, Kinder betreut und von Nachbarn erkannt wird, bleiben: ,[...] ich bin überzeugt, daß man hier in meiner Heimat weit mehr meine Blumen als meine Schriften kennt und bewundert" (BUROW 1857:83).

Otto Roquette schildert dagegen ein ziemlich genaues Bild der Stadt in den 30er Jahren des 19. Jhd.s. Obwohl er kein idealisiertes Stadtbild kreiert, hat ein Teil der Ausführungen einen äußerst positiven Charakter:

Bromberg war schon damals eine hübsche, belebte Stadt, und viele architektonische Altertümer gaben mir zum erstenmale den Anblick von historischem Herkommen. [...] Umgeben von leichten Hügelreihen, zum Theil bewaldet, zum Theil mit neuen Anlagen bepflanzt, von dem raschen Flusse, der Brahe, durchflossen, bot sie schon dem Anblick manches Ungewohnte und Angenehme. Ein lebhafter Verkehr von Krähnen aller Art, besonders Getreideschiffen, zeigte geschäftliche Thätigkeit, gehoben durch große Mühlwerke, welche innerhalb der Stadt hier und dort sogar einen malerischen Anblick boten. Vor allem luden die parkartigen und baumreichen schönen Anlagen, Stunden weit am Kanal, der die Brahe mit der Netze verbindet, zu Spaziergängen ein. Innerhalb der Stadt aber gab es ein vielbewegtes Leben und bürgerlichen Reichtum von alten Handelshäusern. (RoQUETTE 1894:55)

Der Tag der Ankunft in Bromberg und der Ausblick aus den Fenstern der ersten Wohnung am Friedrichplatz werden in der Lebensgeschichte folgendermaßen geschildert:

Der umfangreiche Marktplatz, umschlossen von sauberen Häusern, damals alle noch mit Giebeln versehen, zwischen welchen auf der linken Seite sich eine stattliche Kirche mit zwei Thürmen erhob [die Jesuitenkirche - E. N.]. Beide Thürme trugen damals noch ihren aufgestuften Helmschmuck, welchen ein verheerender Sturm ihnen später [1848 - E. N.] raubte. Aus unseren Fenstern sahen wir gerade 
Bromberg in den Werken deutschsprachiger Schriftsteller

aus in die Brückenstraße, erkennten die Brahebrücke selbst, drüber hinaus alte, einst klösterliche Gebäude [das Karmeliterkloster - E. N.] und das Stadttheater. Alles machte auf die Ankömmlinge den günstigsten Eindruck. (ROQUETTE 1894:54f.)

Als sich die Gelegenheit und Möglichkeit bot, eine neue, größere Wohnung, umgeben von einem Garten und einer Kastanienallee zu beziehen, entschließen sich die Eltern, näher an die Brahe in eine Wohnung zu ziehen, die von dem Gehöft der ehemaligen Zuckersiederei umschlossen war. Die Bedeutung der in Bromberg verbrachten Kindheit determiniert darüber hinaus im Falle Roquettes die Atmosphäre im Königlichen Gymnasium, das er besucht und das, wie er schreibt:

[...] in einem wissenschaftlichen und pädagogischen Verfall war, den nur diejenigen nicht erkannten, welche an nichts Besseres gewöhnt waren, oder nicht gelten lassen wollten, wenn Sie durch die Verbesserung etwas zu verlieren hatten. Obgleich eine deutsche Lehranstalt, wies das Gymnasium unter den Lehrern eine Reihe von Polen auf, welche die deutsche Sprache in einer Weise radebrechten und misshandelten, dass ihre Ausdrucksweise nur dem Eingeborenen oder lange daran gewöhnten verständlich sein konnte, nicht aber dem aus der Ferne zugewanderten [...]. Von einer wissenschaftlichen Vorbildung war bei ihnen nichts zu finden. Ihr Ton und Wesen zeigte eine unglaubliche Rohheit. Die älteren deutschen Lehrer waren durch langjährigen Schlendrian eingerostet, oder sie trieben es den Polen gleich, während das Haupt der Schule, ein uraltes schwaches Männchen, nicht die Energie hatte, der um sich greifenden Zerrüttung der Anstalt zu steuern. (ROQUETTE 1894:86f.)

Die Eltern beschweren sich beim Ministerium wegen der am Gymnasium über ihren Sohn verhängten Leibesstrafen. Dies hat aber nur eine Zuspitzung des Konflikts zur Folge. Die einzige Lösung ist der Schulwechsel. Otto absolviert schließlich das Gymnasium in Frankfurt an der Oder unter der Obhut seines Großvaters. Der Dichter erinnert sich an den Abschied von Bromberg recht sachlich. Der Ich-Erzähler fasst diese Lebensphase folgendermaßen zusammen: „Außer den Eltern und Geschwistern und dem guten Serno [dem Pastor E.N.] verließ ich in Bromberg damals nichts, was mir von Wert gewesen wäre“ (ROQUETTE 1894:104). Der Dichter arbeitet jedoch mit Gegensätzen. Einerseits übt er harte Kritik an den ,grauenhaften“ Schulverhältnissen und an den Lehrern, andererseits schildert er ein sicheres Zuhause und seine künstlerisch, intellektuell und gesellschaftlich aktiven Eltern, die mit namhaften Persönlichkeiten der Bromberger Intelligenz verkehren, indem sie regelmäßig Gesellschaftstreffen organisieren und die intellektuelle Elite der Stadt einladen, darunter den Thorner Schriftsteller Bogumił Goltz (geb. 20.3.1801 in Warschau, gest. 12.11.1870 in Horn) und seinen Bruder, Gymnasialprofessor Hein- 
rich Theodor Rötscher (geb. 20.9.1803 in Mittelwalde, gest. 9.4.1871 in Berlin) mit seiner Frau. Letzterer war schon damals bekannt für seine Arbeiten in Philosophie und Kunstgeschichte. Ferner nennt er den Geheimrat Johann Ferdinand Daniel Neigebaur (geb. 24.6.1783 in Schlesien, gest. 22.3.1866 in Breslau), Albert Schulz (Ps. San Marte, geb. 8.5.1802 in Schwedt, gest. 3.6.1893 in Magdeburg) und nicht zuletzt den jungen Sänger Gustav GrabenHoffmann. Diesen Treffen wird in der Geschichte seines Lebens viel Platz eingeräumt. Über die Musikkultur der Bromberger äußert der Erzähler:

Welch einen Reichtum an musikalischen Kräften man damals in Bromberg beisammen hatte, beweist die Tatsache, dass neben dem öffentlichen Gesangverein, welcher Oratorien aufführte, ein Opernverein gegründet werden konnte, der in Gemeinschaft mit der Liedertafel gute Opern, welchen man im Theater nicht leicht begegnete (zumal man es nur etwa drei Monate im Jahre hatte), einübte, um sie dann als Konzerte aufzuführen. (RoQUETTE 1894:79)

Damit schreibt er gegen die geläufigen Stereotype an, dass Bromberg keine intellektuelle Elite hatte. „Als Sitz der Regierung und einer starken Garnison umfaßte Bromberg eine große Gesellschaft, durcheinandergeweht aus allen Himmelsgegenden, in welcher viel Bildung und geistiges Leben herrschte.“ (ROQUETTE 1894:55)

Für Leontine von Winterfeld-Platen war Bromberg für etwa sieben Jahre (1901-1908) nur eine neue Heimat, weniger bedeutend als z. B. Remlin in Mecklenburg, wo sie am liebsten bei den Großeltern oder ihrem Onkel die Sommerferien verbrachte oder später Ludwigslust, wo sie mit ihrem Mann lebte. In Bromberg lässt sie sich zur Lehrerin ausbilden. Bromberg wird sich aber an sie erinnern, als im Stadttheater die Uraufführung ihres Stücks Sigelind geplant wird. Die Verfasserin wird zu diesem Anlass im Februar 1914 mit ihrem Mann nach Bromberg eingeladen, um den Proben beizuwohnen. ${ }^{11}$

In Dies wilde Jahrhundert von A.E. Johann ist die Stadt viel länger präsent, bis etwa 1920. Danach findet der Erzähler keine Gelegenheit mehr, nach Bromberg zurückzukehren. In seltenen Schilderungen der Topographie erscheint Bromberg als eine ,schöne und ansehnliche“ Stadt (JOHANN 1989:170), als Ort „,mittlerer Größe im ehemaligen deutschen Osten“ (JOHANN 1989:14). Hier absolviert der Ich-Erzähler das Realgymnasium. Ihn faszinieren typische Knabenerlebnisse, die geschickt in Bromberger Realien angesiedelt

$11 \quad$ Bromberger Tageblatt 45 (1914); Ostdeutsche Presse 45 (1914). Bromberger Theaterbrief (1914). In: Aus dem Posener Lande. Monatsblätter für Heimatkunde, Dichtung, Kunst und Wissenschaft des deutschen Ostens (1914), $185 \mathrm{f}$. 
sind. Es ist ein großes Erlebnis, als das erste Automobil nach Bromberg kommt, das dem Sanitätsrat Dietz gehört. Dr. Dietz' Mercedes taucht in Bromberg ungefähr 1905 oder 1906 auf und sollte nicht im Stall, sondern in der Garage untergebracht werden (JOHANN 1989:74). Den bisherigen Kutscher läßt Dr. Dietz in Berlin zum Chauffeur umschulen. Das war ,das erste richtige Automobil hier bei uns im Osten an der russischen Grenze! Das muß man gesehen haben!“ (JOHANN 1989:74) Indem der Umzug der Eltern in eine neue Wohnung geschildert wird, spricht der Erzähler die Lebens- und Wohnbedingungen der preußischen mittleren Beamten in der deutschen Ostmark an:

In der Rinkauer-Straße hatten wir uns nicht sehr wohl gefühlt. Meine Eltern hatten sich durch die Geräumigkeit der Zimmer und gewiß auch durch eine verhältnismäßig niedrige Miete verführen lassen - Schmalhans war ständig bei uns Küchenmeister, ein preußischer Beamter konnte , keine großen Sprünge machen'. [...] Die von Staats wegen zum Beamten-Dasein mitgelieferte Ehre bildete den Ausgleich und Zuschuß zu dem knappen Gehalt. Aber die Wohnung hatte über dem großen Laden, den bescheidenen Privaträumen und der ausgiebigen Werkstatt mit Lagerkammern und Ställen des Hausbesitzers, eines polnischen Schlächtermeisters, gelegen, so dass wir den Lärm und die Gerüche aus der Fleischerei ständig auch bei uns in der Wohnung hatten. (JOHANN 1989:14f.)

Der Umzug in die neue Wohnung verändert jedoch radikal die Lebensbedingungen der Familie Wollschläger.

In der Konrad-Straße, auf der obersten Etage eines neugebauten dreistöckigen Hauses, würden wir es, was am Familientisch reichlich erörtert wurde, nicht nur vornehmer und ruhiger, sondern vor allem auch komfortabler haben mit einem für die damalige Zeit noch weiterhin unerhörten speziellen Badezimmer mit Wanne, Badeofen, feudalem Klo und Wasserspülung, dazu außer dem Holz- und Kohleherd auch noch einem ,Gaskocher' in der Küche, überdies mit einem großen Balkon, von dem man weit ins Land hinausblicken konnte. (JOHANN 1989:14f.)

Genauso beeindruckend berichtet er davon, dass es eines Tages in seinem Elternhaus in allen Zimmern elektrisches Licht gab, das alle anderen Vorzüge übertraf. „Alles, was sonst noch von der neuen Wohnung gefabelt wurde, bedeutete mir nicht viel. Aber wie wohl das Licht aus Draht und Birne flösse, das ließ mich über Nacht nicht schlafen." (JOHANN 1989:14f.) In anderen Zusammenhängen werden in Dies wilde Jahrhundert noch konkrete Orte genannt: die Danziger-, Rinkauer-, Konrad,- und Bahnhofstraße. In der Wilhelmstraße befindet sich die Gasanstalt. Das Lokalkolorit spielt jedoch bei A.E. Johann eine untergeordnete Rolle, es ist nur Hintergrund für die Erlebnisse des heranwachsenden Jungen, der von Technik, Erfindungen und Entdeckungen fasziniert ist. Als manche technischen Neuerungen schon in Bromberg einsetzbar sind, fin- 
det die Begeisterung kein Ende. Bromberg ist bei Johann zum letzten Mal in den Jahren des Ersten Weltkrieges präsent, als halbe Knaben an die Front geworfen werden. Der Ich-Erzähler hatte damals viel Glück, denn nach wenigen Wochen Front ist er lazarettreif geworden. Im Bromberger Lazarett ,herrschte so etwas wie Frieden, denn Luftangriffe mit ,Bombenteppichen“ gab es im Ersten Weltkrieg noch nicht, und die Russen hatten bis auf weiteres jede Bedrohlichkeit verloren, waren vielmehr mit sich beschäftigt und ihrer Großen Revolution“"(JOHANN 1989:190).

Alle genannten autobiographischen Texte vermitteln somit die Kenntnis der Verhältnisse unter den Beamtenfamilien in den Städten der Provinzen Posen und Westpreußen. Sie sind zugleich ein verlässliches Zeugnis der deutsch-polnischen Beziehungen, und diese beiden nationalen Elemente muss man auch vor Augen haben, wenn man an Bromberg in der Vergangenheit denkt. Das allägliche Leben ist schließlich auch davon geprägt, und dies anzusprechen muss der Vollständigkeit wegen getan werden. Alle Autoren vermeiden die Unterstreichung des deutschen oder polnischen Charakters der Stadt. In Versuch einer Selbstbiographie wird das Polenthema außer Acht gelassen. ${ }^{12}$ Bei Otto Roquette wird das deutsch-polnische Nebeneinander nur kurz erwähnt. Den verstreuten Informationen ist zu entnehmen, dass die Eltern des Schriftstellers in den 30er Jahren des 19. Jhd.s keine Kontakte mit polnisch sprechenden Stadtbewohnern pflegen. Polnische Lehrer werden stark kritisiert und in Bezug auf die Polen aus Krotoschin, wo die Familie früher gelebt hat, gebraucht der Ich-Erzähler den abwertenden Begriff „Polacken“. Die nationale bzw. staatliche Zugehörigkeit der Stadt hat jedoch nur eine drittrangige Bedeutung. Leontine von Winterfeld-Platen schenkt der polnischen Thematik in beiden Bänden ihrer Lebenserinnerungen auch keine Aufmerksamkeit. Eine Ausnahme stellt aber A. E. Johann dar. In Dies wilde Jahrhundert wird kurz vor dem Ausbruch des Ersten Weltkrieges in der Atmosphäre der Mobilmachung im Gespräch des Ich-Erzählers mit dem Freund Kurt Folgendes gesagt:

Stell dir das doch mal vor, Mensch: hier die Russen und im Westen die Franzosen und die Engländer und wer weiß, wer noch! Da sitzen wir ja schön in der Zange

12 Unter dem Gesichtspunkt der polnischen Thematik wurde das gesamte Schaffen von Julie Burow eingehend von JeRZY STANISŁAWSKI (1980:161-173) untersucht. Anders als im Versuch einer Selbstbiographie ist die Darstellung der Polen dort sehr präsent und durchweg positiv. So schildere die Schriftstellerin die Polen und ihren Befreiungskampf mit aufrichtiger Sympahie. Gleichzeitig werde die preußische Behörde stark angegriffen. 
Bromberg in den Werken deutschsprachiger Schriftsteller

und müssen nach zwei Seiten fechten. [...] Nach zwei Seiten zugleich kann man sich nicht richtig wehren. Das weißt du doch auch! Und ob ich das wußte! Ich war zwar aus dem entsprechenden Alter schon heraus, hatte aber in jüngeren Jahren in vielen wilden Schlachten reichlich Erfahrungen sammeln können und hatte mir mehr als einmal in Bandenkämpfen (Schule gegen Schule, Polen gegen Deutsche, Katholiken gegen Protestanten, Schröttersdorfer gegen Bleichfelder) blutige Blessuren eingehandelt. (JOHANN 1989:182)

Zum Waffenstillstand am 15.12.1917 zwischen dem Deutschen Reich und Russland schreibt der Ich-Erzähler:

Wir im deutschen Osten brauchten also vorderhand die Russen nicht zu fürchten. Statt dessen regten sich in der preußischen Provinz Posen, dem früheren Großpolen, und in Oberschlesien die Polen, deren sehr verständliche Sehnsucht nach einem eigenen Staat zwischen Deutschland und Russland in den rund 125 Jahren ihrer Staatenlosigkeit nie erloschen war. (JOHANN 1989:190f.)

Die deutsch-polnische Thematik wird im gesamten Buch von Johann auf die obigen Aussagen reduziert. Dagegen ist viele Jahre früher, im ersten Band der autobiographischen Radmacher-Trilogie Im Strom (JoHANN 1942), von den deutsch-polnischen Beziehungen viel mehr zu lesen. In jenem Band läßt sich um 1918/1920 deutlich eine gespannte Atmosphäre zwischen den Vertretern der beiden Nationalitäten feststellen. Sie wird jedoch nicht direkt vermittelt, sondern sie wird anhand der psychischen Verfassung des preußischen Beamten, d.h. des Vaters des Protagonisten, geschildert, als sich in der Stadt 19181920 die politische Lage deutlich zuspitzt. Nach dem Ersten Weltkrieg nimmt Familie Wollschläger Abschied von der polnisch gewordenen Heimat. Etwa 32.000 Deutsche verlassen damals die Stadt (BISKUP 1999:409). Man kann von massenhafter Abwanderung der Deutschen sprechen. Der Anteil der Deutschen an der Gesamtbevölkerung in Bromberg sinkt von $80 \%$ im Jahre 1919/1920 auf 17\% bis 1922 (BISKUP 1999:407). Der Verlust der Heimat im Osten hat seinen Vater stark verwundet. Er hing jedoch nicht an der Stadt. Er hatte eher

[...] Hoffnung und Sehnsucht, als Pensionär endlich einmal in Hamburg oder Bremen die großen Schiffe zu sehen und vielleicht auf Sylt oder Borkum die Brandung des freien Meeres brausen zu hören und die Wellen ans Ufer rollen und schäumen zu sehen - dieser in einem langen Leben des Dienstes für geringes Gehalt und kümmerlichen Urlaub nie zur Ruhe gekommene Wunsch ist ihm nicht erfüllt worden. (JOHANN 1989:138)

Leider ,ist er wenige Wochen, bevor er das Pensionsalter von fünfundsechzig Jahren erreichte, an einer akuten Krankheit gestorben“ (JOHANN 1989:138). 


\section{Schlussfolgerungen}

Der Versuch der Analyse des in den autobiographischen Schriften nur kurz skizzierten Bildes der Stadt und ihrer Bewohner könnte sicher nuancenreicher ausfallen, doch sind die Forschungsergebnisse über die Literatur aus / über Bromberg noch unzulänglich. Erst seit einigen Jahren ist das Thema in Mode. Keiner der genannten Autoren wagt in den autobiographischen Werken ein ganzheitliches Panorama der Stadt, der Verhältnisse und des Alltags in Bromberg darzustellen. Das war auch nicht ihre Absicht. Bromberg bedeutet in ihren Texten nicht viel mehr als ein Wohnort, eine Adresse mit einigen vertrauten Nachbarn und Straßen. Weder Julie Burow, Otto Roquette, Leontine von Winterfeld-Platen noch A.E. Johann sind bekanntlich Autoren, die aus regionaler Erfahrung schreiben. Sie waren mit der Stadt nur in einem bestimmten Moment verbunden, was schon ausreichend war, um sie in die Liste der sogenannten Lokalgrößen aufzunehmen. Bei Otto Roquette und A.E. Johann wird schon in gewisser Hinsicht auf das Werden der Stadt eingegangen, das mittels der Texte wiederentdeckt und wahrgenommen wird. Julie Burow und Leontine von Winterfeld-Platen bleiben in ihrem Verhältnis zur Stadt distanzierter. Im Falle von Julie Burow kann man sagen, dass sie die Barriere des Privaten nicht überwindet. Die fiktionalen Werke der genannten Schriftsteller erfüllen leider nicht das Kriterium der regionalen Literatur über Bromberg. Die Bromberger Thematik, die Bindung an die Stadt, werden in den ausgewählten Texten nicht verleugnet. Sie enthalten schon gewisse Landschafts- und Alltagsbeschreibungen, doch das ist noch lange kein Symptom des Zugehörigkeitsgefühls ihrer Autoren. Sie werden vom Beamtenschicksal geprägt mit allen seinen Folgen. Sie erlauben sich aus diesem Grunde wohl nicht eine lebendigere Beziehung zu der Stadt herzustellen, was deutlich zu spüren ist. Bromberg ist letztendlich doch etwas mehr als nur eine Adresse. Es ist auch die Erinnerung an das Gefühl der Geborgenheit im Elternhaus. Wäre es im Zusammenhang damit sinnvoll, die Diskussion über die Arbeit des Gedächtnisses, die Rolle der Raumverhältnisse, in unserem Fall der Kindheitsräume, gleichzeitig mit der Frage aufzunehmen, was dieser Raum bedeutet?

Das Vorhandensein von topographischen Elementen der Stadt in den Autobiographien ist schließlich umso bedeutender, je jünger die Autoren waren, während sie in Bromberg wohnten. Im Falle von Otto Roquette und A.E. Johann kann man von einer recht großen Sättigung ihrer Texte mit Bromberger Elementen sprechen, wodurch das skizzierte Stadtbild glaubwürdig wird. Die urbanen Elemente spielen bei Julie Burow und Leontine von Winterfeld-Platen keine Rolle. Aus ihren Texten erfährt der Leser kaum etwas über das Aussehen 
Bromberg in den Werken deutschsprachiger Schriftsteller

der Stadt. Doch das Stadtbild muss nicht unbedingt etwas Sichtbares darstellen. Es geht weniger um die Fülle der Details, sondern eher um einen nachhaltigen Eindruck, den der Text auf den Rezipienten macht. Die entsprechend gewählten und zusammengestellten Einzelheiten dienen der Vorstellung einer Ganzheit, wodurch in der Erinnerung ein Bild der provinziellen Städte entsteht, in denen man ähnlich wie in Bromberg lebte. Da schließlich Julie Burow, Otto Roquette, Leontine von Winterfeld-Platen und A.E. Johann mit unterschiedlichen Orten verbunden waren, muss auch noch berücksichtigt werden, dass jeder dieser Orte seinen Anspruch auf diese Autoren erheben darf. Julie Burow war mit Ostpreußen, Elbing und Danzig verbunden. Ihr Name ist auch in den dortigen Schriftstellerverzeichnissen $\mathrm{zu}$ finden (ORŁOWSKI 2003:32f.). Otto Roquette ist vor allem ein Darmstädter, und weil er in Krotoschin geboren ist, gilt er auch als Dichter aus dieser Ortschaft. Leontine von Winterfeld-Platen definiert man in der Regel als eine Vielschreiberin aus Mecklenburg (WIEHLE 1999:189; BRUN 1996:15, 2005:1). Außerdem ist auch Danzig berechtigt, sie durch den Roman Faust über Danzig (1930) als Schriftstellerin, die über Danzig schreibt, zu klassifizieren (ANDRZEJEWSKA / ANDRZEJEWSKI 1998:174). A.E. Johann ist eigentlich ein Weltbürger mit besonderem Bezug zu Kanada. Obwohl alle genannten Autoren nicht als ausgesprochene ,Bromberger Schriftsteller' betrachtet werden können, haben sie trotzdem durch ihre Autobiographien einen wichtigen Beitrag zur Stadt- und Regionalforschung Brombergs geleistet.

\section{Quellen}

BuRow, JuLIE (1857): Versuch einer Selbstbiographie. Leipzig/Prag.

JohANN, A[LFRED] E[RNST] [WoLLENSCHLÄGER] (1942): Im Strom. Berlin.

- (1989): Dies wilde Jahrhundert. München/Wien.

Roquette, Otto (1894): Siebzig Jahre. Geschichte meines Lebens. 2 Bde. Darmstadt.

WinTERFELD-PlATEN, LeONTINE von (1930): Faust über Danzig. Schwerin.

- (1957): Lebenserinnerungen. Stuttgart.

\section{Literatur}

ANDRZEJEWSKA, EWA / ANDRZEJEWSKI, MAREK (1998): Literatur und literarisches Leben in Danzig (1919-1939). In: Studia Germanica Gedanensia 5:169-183.

BANACH, WOJCIECH (2001): Czas odnaleziony. Bydgoszcz na starej pocztówce 18941945. [Die wiedergefundene Zeit. Bromberg auf alten Ansichtskarten 1894-1945]. Bydgoszcz. 
Biskup, Marian (ed.) (1999): Historia Bydgoszczy. [Die Geschichte Brombergs]. 2 Bde. Bydgoszcz.

Bromberger Tageblatt 45 (1914).

Bromberger Theaterbrief (1914). In: Aus dem Posener Lande. Monatsblätter für Heimatkunde, Dichtung, Kunst und Wissenschaft des deutschen Ostens (1914):185f.

Bromberger Wochenblatt 81 (1852).

BRun, Hartmut (1996): Wußten Sie, daß... In: Ludwigsluster Stadtanzeiger. Amtliches Bekanntmachungsblatt der Stadt Ludwigslust 21:15.

- (2005): Leontine aus altem Adel. Literarische Merk-Würdigkeiten aus Mecklenburg. In: LeuchtTurm: das norddeutsche Magazin im Aufbruch 3:1.

DerendA, Jerzy (2007): Piękna stara Bydgoszcz. [Das schöne, alte Bromberg]. Bydgoszcz.

FRIEDRICHS, ELISABETH (1967): Literarische Lokalgrößen 1700-1900. Verzeichnis der in regionalen Lexika und Sammelwerken angeführten Schriftsteller. Stuttgart.

KURZ, HEINRICH (1872): Geschichte der neuesten Literatur von 1830 bis auf die Gegenwart. Leipzig.

Laskowsky, Paul (1927): Dichter des Posener Landes. In: Braun, Fritz / LüdtKe, FRANZ / MÜLLER-RÜDERSDORF, WILHELM (eds.): Entrissene Ostlande. Ein Heimatbuch. Leipzig, 178-188.

LeJeune, PhILIPPE (2001): Wariacje na temat pewnego paktu. O autobiografii. [Der autobiographische Pakt]. Kraków.

Loew, Peter Oliver (2000): Konstruktion und Rekonstruktion des Danziger Alltags vergangener Zeiten im 19. und 20. Jahrhundert. In: ANDRZEJEWSKI, MAREK (ed.): Beiträge zum Alltagsleben. Danzig, Bremen und die Antike. Gdańsk, 101f.

Machatscheck, Hans (1930): Der Ostdeutsche Otto Roquette und das Bromberg seiner Zeit. In: Deutsche Schulzeitung in Polen 19/20:285-289.

Minde-Pouet, Georg (1911): Die Provinz Posen in der modernen deutschen Dichtung. In: Korrespondenzblatt des Gesamtvereins der deutschen Geschichts- und Altertumsvereine 6/7:273-283.

NowIKIEWICZ, ELŻBIETA (2002): Wspomnienie o salonie rodziny Roquette. [Erinnerung an den Salon der Familie Roquette]. In: Kronika Bydgoska XXIII:302-312.

OrŁowsKi, Hubert (2003): Za górami, za lasami ... O niemieckiej literaturze Prus Wschodnich 1863-1945. [Hinter den Bergen, hinter den Wäldern... Zur deutschen Literatur in Ostpreußen]. Olsztyn.

Ostdeutsche Presse 78/79 (1883).

- 45 (1914).

Ostdeutsche Rundschau 14/15 (1909).

POŁCZYŃSKA, EdYTA (1988): Im polnischen Wind. Beiträge zum deutschen Zeitungswesen, Theaterleben und zur deutschen Literatur im Großherzogtum Posen 1815-1918. Poznań (=Seria Filologia Germańska 32). 
Bromberg in den Werken deutschsprachiger Schriftsteller

PoŁczyńska, Edyta / Wojtczak, Maria (1996): Die Provinz Posen in der deutschen Literatur um die Jahrhundertwende. In: Convivium:83-106.

ROSNER, EDMUND (1975): Regionalizm w nauczaniu języka polskiego w szkole średniej. [Regionalismus im Polnischunterricht in der Oberschule]. Warszawa.

SAMEL, WIELFRIED (1993): Abteilung für Literatur. Vor neunzig Jahren in Bromberg gegründet. In: Bromberg 101:9f.

STANISŁAWSKI, JERZY (1980): Julia Burow Pfannenschmidt i jej twórczość w aspekcie problematyki polskiej. [Julie Burow Pfannenschmidt und ihr Schaffen im Hinblick auf die deutsch-polnische Problematik]. In: Studia Historia-Slavogermanica 9:161-173.

Wiehle, MARTIN (1999): Altmark-Persönlichkeiten. Biographisches Lexikon der Altmark, des Elbe-Havel-Landes und des Jerichower Landes. Oschersleben (=Beiträge zur Kulturgeschichte der Altmark 5), 189.

WoJTCZAK, MARIA (1998): Literatur der Ostmark - Posener Heimatliteratur (18901918). Poznań.

- (1999): Literatur der Ostmark - „Posener Heimatliteratur“ (1890-1918). In: Zeszyty Naukowe Wyższej Szkoty Pedagogicznej w Bydgoszczy. Konfiguracje/Konfigurationen 4:94-104.

- (2001): Ostmarkenliteratur. Prowincja poznańska w literaturze niemieckiej lat 18901918. [Die Provinz Posen in der deutschen Literatur in den Jahren 1890-1918]. Poznań. 